\title{
Using Real-Time fMRI to Control a Dynamical System by Brain Activity Classification
}

\author{
Anders Eklund ${ }^{1,2}$, Henrik Ohlsson ${ }^{3}$, Mats Andersson ${ }^{1,2}$, Joakim Rydell ${ }^{1,2}$, \\ Anders Ynnerman ${ }^{4,2}$, and Hans Knutsson ${ }^{1,2}$ \\ 1 Div. of Medical Informatics, Linköping University, Sweden \\ \{andek, matsa, joary, knutte\}@imt.liu.se \\ 2 Center for Medical Image Science and Visualization (CMIV) \\ 3 Div. of Automatic Control, Linköping University, Sweden \\ ohlsson@isy.liu.se \\ ${ }^{4}$ Div. for Visual Information Technology and Applications \\ Linköping University, Sweden \\ andyn@itn.liu.se
}

\begin{abstract}
We present a method for controlling a dynamical system using real-time fMRI. The objective for the subject in the MR scanner is to balance an inverted pendulum by activating the left or right hand or resting. The brain activity is classified each second by a neural network and the classification is sent to a pendulum simulator to change the force applied to the pendulum. The state of the inverted pendulum is shown to the subject in a pair of VR goggles. The subject was able to balance the inverted pendulum during several minutes, both with real activity and imagined activity. In each classification 9000 brain voxels were used and the response time for the system to detect a change of activity was on average 2-4 seconds. The developments here have a potential to aid people with communication disabilities, such as locked in people. Another future potential application can be to serve as a tool for stroke and Parkinson patients to be able to train the damaged brain area and get real-time feedback for more efficient training.
\end{abstract}

\section{Introduction}

Despite the enormous complexity of the human mind, fMRI, functional Magnetic Resonance Imaging, techniques are able to partially observe the state of a brain in action. In conventional fMRI, the experiment is performed and the data is analyzed afterwards to calculate a level of brain activity for each voxel. In realtime fMRI, the data is analyzed directly and the result from the analysis can be used to change the stimulus presented to the subject, see figure 1. The brain state can be interpreted by a computer and the setup is then often called a brain computer interface (BCI).

Brain computer interfaces first used electroencephalography (EEG). Some consider EEG to be superior to fMRI since it has a much higher temporal resolution. fMRI, however, has a higher spatial resolution than EEG. Even if the 


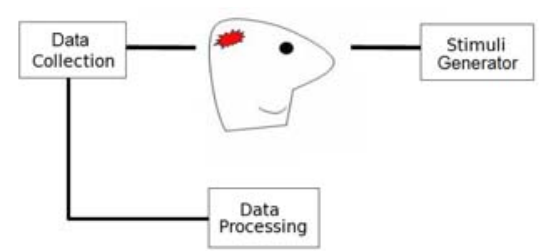

(a) A conventional fMRI experiment setup. The data is collected during the stimulus paradigm and is analyzed after the experiment to get a measure of brain activity in each voxel. The analysis can be made in real-time but is not used to change the stimulus given to the subject.

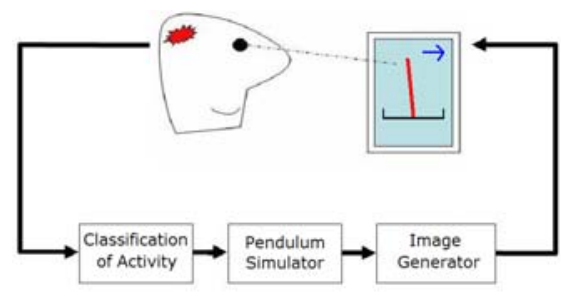

(b) The experiment setup for a biofeedback loop using real-time fMRI. The subject sees the red pendulum. The activity for each new volume of data is classified and sent to the pendulum simulator. The blue arrow shows the current classification of the brain activity.

Fig. 1. The experiment setups for conventional fMRI and bio-feedback using real-time fMRI. In real-time fMRI we can achieve a bio-feedback loop, by using the result from the real-time analysis to change the stimulus, where the subject can control a dynamical system.

EEG signal is sampled with $5 \mathrm{kHz}$, most of the brain activity is below $100 \mathrm{~Hz}$ and an inverse problem has to be solved in order to reconstruct the spatial position of the brain activity. In most clinical applications, 19 recording electrodes are used according to the international 10-20 system [1. The usable bandwidth of a normal clinical EEG application is thus 19 channels at $100 \mathrm{~Hz}$, resulting in 1900 sample points per second. In our fMRI setup, we have 9000 channels (brain voxels) at $1 \mathrm{~Hz}$, resulting in 9000 sample points per second. Faster scanning schemes using compressed sensing [2], can in the future increase the temporal resolution of fMRI. With stronger MR scanners it is also becoming easier to detect the small initial dip of the BOLD signal [3], and thus making it unnecessary to wait for the peak of the delayed BOLD response.

We have tested our BCI with a dynamical pole balancing experiment. In the experiment, the subject was given the possibility to move an inverted pendulum. The pendulum could be pushed to the left or right by activating the parts of the motor cortex associated with activity of the left and right hand. Similar projects have been presented in. [4] and [5]. Laconte et al. uses a similar setup in [5] but the arrow that they control can not be considered to be a dynamical system that changes by itself. The dynamical properties of the inverted pendulum make our setup a more challenging problem. We have to interpret the desire of the subject and set out a control signal moving the pendulum as often as once a second to have a chance to handle the fast dynamics of the pendulum.

The area of real-time fMRI is relatively new compared to conventional fMRI. Laconte et al. [5] were one of the first to perform real-time classification of brain activity. Ohlsson et al. 6] use real-time fMRI to determine where you are looking. deCharms et al. 7] use real-time fMRI for learning a subject to suppress its own pain. Weiskopf et al. [8] use real-time fMRI for self-regulation of local brain activity. An overview of fMRI brain computer interfaces is given by Sitaram et al. [9]. 


\section{Methods}

\subsection{Measuring Brain Activity by Correlation}

In fMRI the objective is to find the intensity and spatial position of brain activity. The estimation of brain activity is based on the fact that the magnetic properties of the blood changes when the neurons demand more oxygen to compensate for their increased activity. The body overcompensates the amount of oxygen sent to the neurons. The signal that is measured in fMRI is called the BOLD signal, where BOLD stands for blood oxygen level dependent.

In conventional fMRI, brain activity is often measured using correlation between the stimulus paradigm and the intensity time series of each voxel. A stimulus paradigm can for example consist of alternating between finger tapping and rest in periods of 20 seconds each. Since the subject is told what to do we calculate the brain activity for each voxel as the correlation with the stimulus paradigm. In order to improve the novel correlation method, several methods have been proposed. The most frequently used is the general linear model (GLM), e.g. Friston et al. [10].

\subsection{Classification of Brain Activity}

In our real-time fMRI setup, the subject acts independently of any paradigms. Thereby we do not have anything to correlate with. The BOLD signal that is detected in fMRI does not occur directly when some brain activity is started but is delayed 3-5 seconds. This is a property of the human physiology that we can not change. This makes it hard to control a system in real-time. In conventional fMRI it does not matter other than that the delay is unknown. Other difficulties in real-time fMRI are that it is harder, compared to conventional fMRI, to detrend the time series of each voxel and that all calculations have to be made in real-time. Detrending is needed since there are drifts and trends in the fMRI data that will corrupt the estimates if not removed [11.

Instead of using correlation, we classify each volume of data to know what the current brain activity is. In this project we classify between left hand activity, right hand activity and rest. A training phase is used to learn how to classify between the different types of brain activity. We used a one layer neural network to classify the brain activity as a first approach since it is easy to implement and to explain and provided sufficient discrimination

\subsection{Neural Networks}

Neural networks 12 are used in many applications to classify data into a number of predetermined classes. Often some informative properties are first extracted from the data instead of using the raw data itself in the classification. Our first approach was to find active voxels in the left and right motor cortex and use them for the classification, to reduce the dimension of the original data. However, it is a known fact that even if the subject is only activating one hand, there will 
be activity in both the left and right part of the motor cortex. Hence it is hard to create a stimulus paradigm for the training phase of the classifier to find voxels that only are active in each state. In the more simple case with only two states, rest and activity, we can find the best voxels, by correlation, to use in the classification.

Neural networks can have arbitrary many layers of perceptrons. A one layer neural network is sufficient if the classes are linearly separable, otherwise at least two layers have to be used in order to classify the data. The best weights $w$ of the discriminant function is derived during the training phase where training vectors and the according correct classifications are presented to the neural network. In our training phase the subject follows a pre-determined stimulus paradigm. We trained the neural network with all the brain voxels in each collected volume, using a spatial mask. Normally 9000 voxels, out of 64000 , were considered to be brain voxels. Each volume was also filtered by a $3 \times 3 \times 3$ Gaussian lowpass filter to make the classifications more robust to motions.

We used a one layer neural network with 3 output nodes. Each output node has a weight vector of dimension 9000 that is achieved during the training phase. In the training phase we used a 240 second long stimulus paradigm consisting of 20 seconds of left activity, followed by 20 seconds of rest and then 20 seconds of right activity, repeated 4 times. A volume of data was collected each second resulting in 240 training vectors, each of dimension 9000, for the neural network.

Since the dimension of the data is 9000 and the number of training vectors is only 240 , we use a linear activation function during the training of the neural network. This means that our neural network is equivalent to using linear regression for classification. If for example tanh would have been used as an activation function instead, the risk of overfitting would have been large since the number of dimensions is much higher than the number of training examples. To make a classification, three scalar products are calculated and the class corresponding to the output node with the highest output, meaning the furthest distance from the decision boundary, is selected. In the real-time phase, each volume was spatially smoothed ( $3 \times 3 \times 3$ Gaussian lowpass filter) and detrended (by removing the mean value calculated 45 seconds backwards for each voxel separately). A control signal (apply a force to the left, right or do nothing) was then computed by applying the, in the training phase computed, neural network. The control signal was used as an input to the simulation of the dynamical pendulum system. The simulation uses the control signal to change the state of the system by applying an according force to the pendulum.

\subsection{Head Movement}

One potential cause of error in fMRI is that the subject may involuntarily move the head in pace with the stimulus paradigm and thereby induce high correlation in voxels on the edge of the brain or outside the brain.

For a neural network it is straight forward to calculate the importance of each voxel for each classification. From this we can eliminate the possibility that the reason that our experiment setup works is due to head movement. 


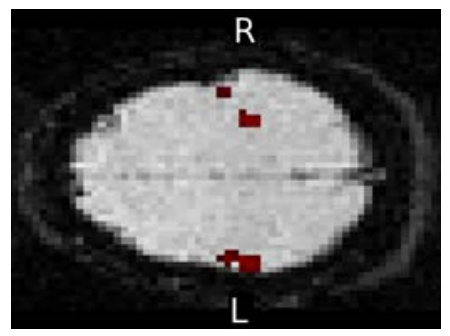

(a) The most important voxels for classification of left hand activity at one timepoint.

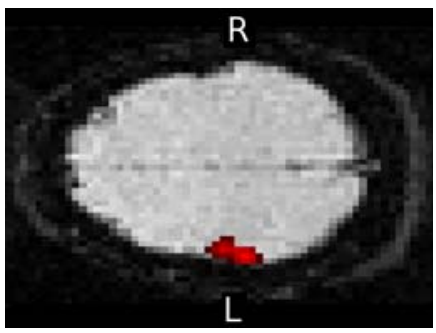

(b) The most important voxels for classification of right hand activity at one timepoint.

Fig. 2. The figures show which voxels that are the most important for classification of brain activity at two timepoints. The importance maps should not be interpreted as activity maps. It is clear that the voxels in the motor cortex, as expected, are the most important. If head movement was the reason that the experiment setup worked, the voxels at the edge of the brain would have been important for each classification. The explanation for the overlap for left and right hand activity classification, which is not present for all timepoints, is that the information that there is no activity in one side of the motor cortex is important for the classification and that bilateral activation of the motor cortex is common (but different for left and right hand activation). We use the radiological display convention and have marked the left and the right side with $\mathrm{L}$ and $\mathrm{R}$.

If the distance to the decision boundary, for a classification at one timepoint $t$, is denoted with $d_{t}$, then the importance $i$ of voxel $v_{n t}$ (voxel $v_{n}$ at timepoint t) can be calcuclated as

$$
i_{v_{n t}}=\frac{w_{n} v_{n t}}{d_{t}}=\frac{w_{n} v_{n t}}{\sum_{k} w_{k} v_{k t}}
$$

where $w_{n}$ is the weight in the neural network for voxel $v_{n}$. Before the calculation is made, one has to make sure that the voxel contributes to the right direction from the decision boundary, i.e. that $w_{n} v_{n t}>0$. If these calculations are made for all the brain voxels, there will be a lot of voxels that are important for some timepoint but not for a set of continuous timepoints. In order to remove the flickering voxels, a median filtering of the time series of the importance value for each voxel can be made. The resulting importance maps are shown in figure 2 , A $3 \times 3$ gaussian lowpass filter has been used to improve the appearance of the importance maps.

\section{Experiment Setup}

The data was acquired using a 1.5 T Philips Achieva MR scanner. The acquisition resolution was $80 \times 80 \times 10$ voxels. Field of view and slice thickness were chosen to obtain a voxel size of $3 \times 3 \times 3 \mathrm{~mm}$. Echo time (TE) was set to $40 \mathrm{~ms}$ and repetition time (TR) was set to $1000 \mathrm{~ms}$. The classification of the brain activity and the simulation of the inverted pendulum was carried out in Matlab on a 
standard laptop. The current state of the inverted pendulum was shown to the subject in a pair of VR goggles.

The resulting angular acceleration was calculated in each iteration and we used a small timestep to calculate the angular velocity and the angle of the pendulum. The simulation of the pendulum was carried out continously and each time we collected a new volume of data (once a second), a classification of brain activity was made and used to set the force applied to the pendulum.

\section{Results}

The subject was able to balance the inverse pendulum both with real activity and imagined activity. When the subject used imagined activity, the pendulum was balanced by only thinking of activating the left or right hand. To justify the success of the controller figure 3 shows the logged angle of the pendulum and the classified activity during the real-time phase. The figure shows that the subject could balance the pendulum for $250 \mathrm{~s}$. The figure also shows the dynamical properties of the pendulum. To validate that this significantly differs from chance, 5000 simulation runs of $800 \mathrm{~s}$ each was performed with the pendulum simulator with random activity. 35 out of 59446 simulated balancing attempts $(0.059 \%)$ were then longer than $250 \mathrm{~s}$.

We also tested the neural network offline with four different datasets, two generated by real activity $(\mathrm{R} 1, \mathrm{R} 2)$ and two generated by imagined activity (I1, I2). All datasets were $240 \mathrm{~s}$ long. The detrending was performed as in the real-time phase. The number of correct classifications, as function of training dataset and evaluation dataset used, is given in table 1 below. A phase based $3 \mathrm{D}$ rigid body motion compensation algorithm was implemented. It did not run in real-time during the experiment but was used offline for validation.

Table 1. Neural network classification performance as function of training dataset and evaluation dataset

\begin{tabular}{|c|l|l|l|l|l|l|}
\hline Training dataset / Evaluation dataset & $\mathrm{R} 1 / \mathrm{R} 2$ & $\mathrm{R} 2 / \mathrm{R} 1$ & $\mathrm{I} 1 / \mathrm{I} 2$ & $\mathrm{I} 2 / \mathrm{I} 1$ & $\mathrm{R} 1 / \mathrm{I} 1$ & $\mathrm{R} 1 / \mathrm{I} 2$ \\
\hline Without motion compensation & $93.7 \%$ & $86.3 \%$ & $86.7 \%$ & $85.8 \%$ & $90.4 \%$ & $83.3 \%$ \\
\hline With motion compensation & $93.4 \%$ & $90.4 \%$ & $86.3 \%$ & $86.7 \%$ & $90.8 \%$ & $83.8 \%$ \\
\hline Training dataset / Evaluation dataset & $\mathrm{R} 2 / \mathrm{I} 1$ & $\mathrm{R} 2 / \mathrm{I} 2$ & $\mathrm{I} 1 / \mathrm{R} 1$ & $\mathrm{I} 1 / \mathrm{R} 2$ & $\mathrm{I} 2 / \mathrm{R} 1$ & $\mathrm{I} 2 / \mathrm{R} 2$ \\
\hline Without motion compensation & $87.5 \%$ & $77.1 \%$ & $90.0 \%$ & $90.0 \%$ & $89.6 \%$ & $91.7 \%$ \\
\hline With motion compensation & $87.1 \%$ & $77.9 \%$ & $89.2 \%$ & $88.8 \%$ & $87.1 \%$ & $91.7 \%$ \\
\hline
\end{tabular}

It is worth to mention that classification with ordinary linear regression performs as well as the far more advanced support vector machines (SVM) approach used by Laconte et al. [5]. 
Classifications of activity (blue stars) and pendulum angle (red line) as function of time

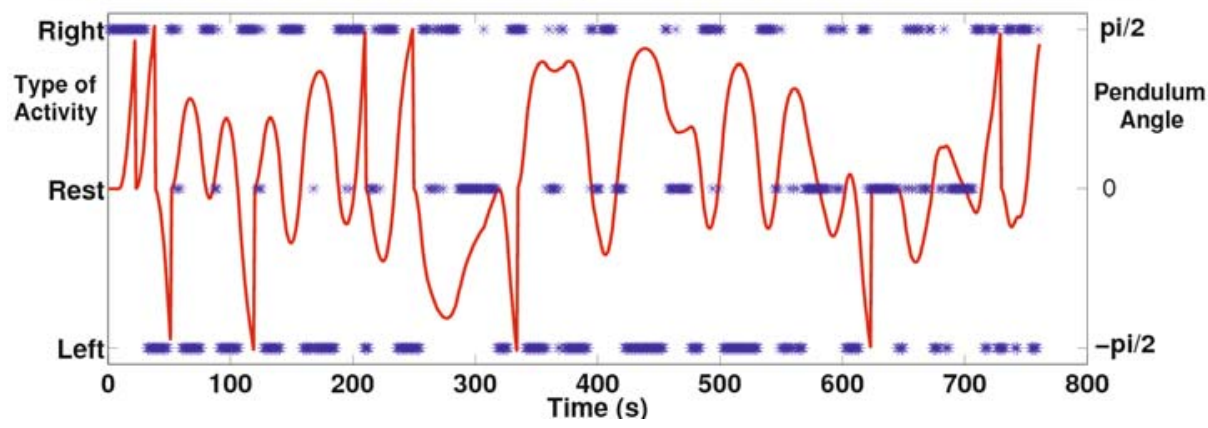

Fig. 3. The figure shows the angle of the pendulum, the red line, and the classifications of activity, the blue stars, as a function of time in the real-time phase. If the angle exceeds $-\frac{\pi}{2}$ or $\frac{\pi}{2}$ the pendulum is restarted and the angle is set to zero, this happened 9 times in this real-time phase. The test subject has to compensate for the delayed BOLD-signal by changing activity a few seconds in advance. It is easy to see that the test subject needed a couple of attempts first to learn the dynamics of the system. The effective force applied to the pendulum depends on the angle of the pendulum as $\cos (\alpha)$. Angle 0 means that the pendulum is standing straight up, $-\frac{\pi}{2}$ means that the pendulum lies along the negative $\mathrm{x}$-axis and $\frac{\pi}{2}$ means that the pendulum lies along the positive $\mathrm{x}$-axis. This means that it is easy to move the pendulum when it is standing straight up, but it takes a long time to straighten it up if the angle is close to $-\frac{\pi}{2}$ or $\frac{\pi}{2}$. If the activity is classified as left activity, a force to the left on the pendulum is applied and the angle of the pendulum decreases. If the activity is classified as right activity, a force to the right is applied and the angle of the pendulum increases. If the activity is classified as rest, no force is applied (other than the gravitational force) and the pendulum continues to rotate in its current angular direction. Between approximately timepoint 350 and 600 in the figure the pendulum was successfully balanced by the subject.

\section{Discussion}

We have presented an fMRI based BCI realization. The human brain and a computer were here linked by fMRI and worked together as a controller of a dynamical system. The dynamical system was made up of an inverted pendulum. The subject had the ability to induce a force by evoking brain activity in the motor cortex. A neural network was trained to separate between left and right hand activity and rest. The subject was able to balance the inverted pendulum both with real activity and with imagined activity. In the future we would like to improve the detection speed of the system. One way to do this is to train the classifier on the transitions between the different states instead of the states them self, as mentioned in [5]. This can be done by looking back at the signal a number of seconds to learn what the different transitions look like, to earlier detect a change of activity. We would also like to increase the bandwidth of the biofeedback loop by including a larger number of different simultaneous activities 
to make the subject able to control a more advanced dynamical system. We also want to make our motion compensation algorithm to run in real-time and compare it to existing techniques $[13$.

The developments here can be used for neuro-scientific investigations 14 and also have a potential to aid people with communication disabilities, such as locked in people [15. Other possible applications are to learn how control your own pain [7] or to serve as a tool for stroke [16] and Parkinson [17] patients to be able to train the damaged brain area and get real-time feedback for more efficient training.

Acknowledgments. This work was supported by the Strategic Research Center MOVIII, funded by the Swedish Foundation for Strategic Research, SSF.

\section{References}

1. Niedermeyer, E., da Silva, L.: Electroencephalography: Basic Principles, Clinical Applications, and Related Fields, 5th edn. Lippincott Williams \& Wilkins (2004) ISBN 0781751268

2. Lustig, M., Donoho, D., Pauly, J.M.: Sparse MRI: The application of compressed sensing for rapid MR imaging. Magnetic Resonance in Medicine 58, 1182-1195 (2007)

3. Yacoub, E., Hu, X.: Detection of early negative response in fMRI at 1.5 tesla. Magnetic Resonance in Medicine 41, 1088-1092 (1999)

4. Yoo, S., Fairneny, T., Chen, N., Choo, S., Panych, L.P., Park, H., Lee, S., Jolesz, F.A.: Brain-computer interface using fMRI: spatial navigation by thoughts. Neuroreport 15, 1591-1595 (2004)

5. Laconte, S.M., Peltier, S.J., Hu, X.P.: Real-Time fMRI Using Brain-State Classification. Human Brain Mapping 28, 1033-1044 (2007)

6. Ohlsson, H., Rydell, J., Brun, A., Roll, J., Andersson, M., Ynnerman, A., Knutsson, H.: Enabling Bio-Feedback Using Real-Time fMRI. In: 47th IEEE Conference on Decision and Control (2008)

7. de Charms, R.C., Maeda, F., Glover, G.H., Ludlow, D., Pauly, J.M., Soneji, D., Gabrieli, J.D.E., Mackey, S.C.: Control over brain activation and pain learned by using real-time functional MRI. PNAS 102, 18626-18631 (2005)

8. Weiskopf, N., Scharnowski, F., Veit, R., Goebel, R., Birbaumer, N., Mathiak, K.: Self-regulation of local brain activity using real-time functional magnetic resonance imaging (fMRI). Journal of Physiology-Paris 98, 357-373 (2004)

9. Sitaram, R., Caria, A., Veit, R., Gaber, T., Rota, G., Kuebler, A., Birbaumer, N.: fMRI Brain-Computer Interface: A Tool for Neuroscientific Research and Treatment. Computational Intelligence and Neuroscience (2007)

10. Friston, K.J., Holmes, A.P., Worsley, K.J., Poline, J.B., Frith, C., Frackowiak, R.S.J.: Statistical Parametric Maps In Functional Imaging: A General Linear Approach. Human Brain Mapping 2, 189-210 (1995)

11. Friman, O., Borga, M., Lundberg, P., Knutsson, H.: Detection and detrending in fMRI data analysis. NeuroImage 22, 645-655 (2004)

12. Haykin, S.: Neural networks - a comprehensive foundation. Prentice-Hall, Englewood Cliffs (1999) 
13. Oakes, T.R., Johnstone, T., Walsh, K.S.O., Greischar, L.L., Alexander, A.L., Fox, A.S., Davidson, R.J.: Comparison of fMRI motion correction software tools. NeuroImage 28, 529-543 (2005)

14. Hollmann, M., Baecke, S., Mueller, C., Bernarding, J.: Predicting Human Decisions in a Social Interaction-Scenario Using Real-Time Functional Magnetic Resonance Imaging (Rt-FMRI), ISMRM (2009)

15. Boly, M., Coleman, M.R., Davis, M.H., Hampshire, A., Bor, D., Moonen, G., Maquet, P.A., Pickard, J.D., Laureys, S., Owen, A.M.: When thoughts become action: An fMRI paradigm to study volitional brain activity in non-communicative brain injured patients. NeuroImage 36, 979-992 (2007)

16. Carey, J.R., Kimberley, T.J., Lewis, S.M., Auerbach, E.J., Dorsey, L., Rundquist, P., Ugurbil, K.: Analysis of fMRI and finger tracking training in subjects with chronic stroke. Brain 125, 773-788 (2002)

17. Wu, T., Hallett, M.: A functional MRI study of automatic movements in patients with Parkinson's disease. Brain 128, 2250-2259 (2005) 